\title{
Deutsch als Fremdsprache für ausländische Studierende und Wissenschaftler ${ }^{1}$
}

\section{Christian Bode}

Für die Einladung zu dieser Tagung danke ich dem Veranstalter herzlich. Gerne nehme ich Gelegenheit, zum Thema "Sprachliche Ausbildung ausländischer Studierender und Wissenschaftler « einige Anmerkungen aus Sicht des DAAD $\mathrm{zu}$ machen. Ich denke, ich darf Ihres Einverständnisses sicher sein, wenn ich das Thema ein wenig anders akzentuiere, als der Wortlaut im ausgedruckten Programm erwarten läßt: Als Laie kann ich zur fachlichen Diskussion wenig beitragen. Mir liegt vielmehr an einer Diskussion der hochschulpolitischen und bildungspolitischen Rahmenbedingungen des Ausländerstudiums in Deutschland und in diesem Rahmen eben auch der Bedingungen und der möglichen Weiterentwicklungen der Sprachausbildung.

Um die sprachliche Ausbildung der ausländischen Studierenden sind bekanntlich die Lehrgebiete DaF bemüht. Ihnen möchte ich für Ihre wichtige und engagierte Arbeit hier mit Nachdruck danken, und ich möchte klarstellen: Die Überlegungen des DAAD haben die Stärkung, nicht etwa die Kritik der Lehrgebiete zum Ziel. Denn Ihre Arbeit ist durch die neue Diskussion um den Studien- und Wissenschaftsstandort Deutschland noch wichtiger geworden. Dies deutlich zu machen ist mir auch deshalb wichtig, weil einzelne programmpolitische Entscheidungen oder Reformüberlegungen des DAAD hier und da als Votum gegen eine anspruchsvolle Sprachausbildung mißverstanden worden sind. Wenn wir die Bedeutung des Englischen als internationale Wissenschaftssprache betonen und uns auch für gezielte Ausbildungsangebote in Englisch aussprechen, wenn wir dem Erwerb deutscher Sprachkenntnisse schon im Heimatland einen hohen Stellenwert beimessen, wenn wir für eine Weiterentwicklung und Flexibilisierung der DSH eintreten und wenn wir schließlich die Entwicklung eines einheitlichen DaF-Tests in Angriff nehmen, dann haben alle diese Maßnahmen eine bedarfsgerechte Sprachausbildung unserer ausländischen Gäste zum Ziel. Bedarfsgerecht heißt dabei eine sprachliche Ausbildung, die den praktischen Anforderungen im Alltag genügt, der Teilhabe am öffentlichen und kulturellen Leben dient und den Anforderungen im Studienoder Forschungsvorhaben gerecht wird. Bedarfsgerecht heißt ferner: kundenfreundlich und dazu wiederum gehören bezahlbare Preise und kalkulierbarer, vertretbarer Zeitaufwand.

1 Bei diesem Beitrag handelt es sich um die überarbeitete Fassung des Vortrages, den der Generalsekretär des Deutschen Akademischen Austauschdienstes (DAAD) am 23. Mai 1997 im Rahmen der 25. Jahrestagung Deutsch als Fremdsprache des FaDaF an der Johannes Gutenberg-Universität Mainz gehalten hat. 
Seit zwei Jahren wird mit großer öffentlicher Aufmerksamkeit über die internationale Konkurrenzfähigkeit und Attraktivität des Studienstandorts Deutschland diskutiert. Der DAAD hat diese Diskussion mit angestoßen, um zur Verbesserung der gegenwärtigen Situation mit beizutragen, nicht um lamentierend das deutsche Hochschulsystem schlechtzumachen. Die Politik hat mit großer Resonanz und prominent reagiert: Die KMK, die HRK, die Minister Rüttgers und Kinkel und die Konferenz der Regierungschefs haben sich des Themas angenommen. Politisch hat das Anliegen zur Zeit durchaus Unterstützung, freilich wird angesichts leerer öffentlicher Kassen nur im Falle einzelner Projekte, nicht aber für den gesamten Hochschulbereich mit zusätzlichen Geldmitteln zu rechnen sein. Die Botschaft der großen Wahlsieger in USA und Großbritannien, die das Bildungswesen an die Spitze ihrer Agenda gesetzt haben, hat sich hierzulande noch nicht herumgesprochen. Wir leisten uns statt dessen einen anderen historischen Rekord: erstmals in diesem Jahr übersteigen die Ausgaben für Arbeitslose mit über 180 Mrd. DM die öffentlichen Gesamtausgaben für Bildung und Wissenschaft.

Es wäre allerdings eine verengte Sichtweise und eine falsche, sogar fatale Zielsetzung, wollten wir an erster Stelle lediglich um mehr Geld argumentieren. Zumindest sollten wir zuerst die Schularbeiten machen, die kostenneutral erledigt werden können. Dabei geht es z. B. um politische und rechtliche Aspekte: Die Einreiseprozeduren sind oft $\mathrm{zu}$ hürdenreich und zu langwierig; wir tun uns $\mathrm{zu}$ schwer mit der Anerkennung im Ausland erbrachter Studienleistungen, von »werben« gar nicht zu reden, wir informieren in den Heimatländern oft noch unzureichend, die Studienstruktur und das System unserer Abschlüsse sind für viele Partnerländer ob ihrer Länge und Unkalkulierbarkeit eher abschreckend als einladend. Und nicht zuletzt haben wir es geschafft, uns den Ruf beizulegen, die schwierigste Sprache der Welt zu sprechen - auch nicht gerade eine Ermunterung für ausländische Gäste.

Wenn wir um Verbesserungen und Erleichterungen bemüht sind, dann ist die Zielsetzung nicht, als Studentenimporteur mit möglichst hohen Zahlen zu imponieren. Ausländische Studierende sind - da es in Deutschland keine Studiengebühren, schon gar keine kostendeckenden gibt - kein materieller, sondern ein bildungspolitischer Gewinn, und dies eben vor allem dann, wenn wir die Guten und die Besten gewinnen. Es geht also darum, für die besten Köpfe, für den begabten Nachwuchs attraktive Studienund Arbeitsbedingungen anzubieten, und diese Form der Zusammenarbeit ist sehr wohl in unserem ureigensten Interesse.

Sie stärkt die Internationalität der Wissenschaft und unserer Ausbildung hierzulande und sie gewährleistet, daß zu den zukünftigen Eliten der Partnerländer auch Personen zählen, die Deutschland aus eigener Anschauung gut kennen und mit den deutschen Belangen vertraut sind. Ich will gar nicht unerwähnt lassen, daß dies auch eine wirtschaftliche Seite hat, die in der gegenwärtigen Standortdebatte besonderes politisches Gewicht bekommt.

Ich will auch das höchst sensible Thema der Scheinstudenten nicht unerwähnt lassen. Es gibt Länder, aus denen so manche Studienbewerber alles andere als ein Studium im Sinn haben. Wer also für erleichterten Zugang zum Studium eintritt, der muß sich auch der Frage nach einer Steuerung bzw. nach einer Auswahl stellen. Nach meiner Meinung müssen die Hochschulen mehr Möglichkeiten erhalten und vor allem auch nutzen, über 
die Zulassung nach fachlichen Qualitätskriterien zu entscheiden. Jedenfalls darf dies nicht ersatzweise über die DSH erfolgen. Diese Prüfung ist sowohl für quantitative als auch für qualitative Steuerung der Ausländerzulassung das völlig falsche Instrument.

Wenn ich nun auf die sprachlichen Anforderungen im einzelnen eingehe, so will ich zunächst betonen, daß wir für den Spracherwerb nicht oder doch nicht ausschließlich das Bild von der Hürde wählen sollten. Für viele Studenten und Wissenschaftler ist es keine zusätzliche, notgedrungen in Kauf zu nehmende Erschwernis, sondern ausdrücklich erklärtes oder gar vorrangiges Ziel, gute Kenntnisse der deutschen Sprache zu erwerben. Und wenn uns, wie anfangs ausgeführt, daran liegt, daß wir guten Nachwuchs so ausbilden, daß er mit Deutschland vertraut wird und auch langjähriger Partner bleibt, dann muß uns natürlich auch daran gelegen sein, daß diese Partner ein wirklich tragfähiges, ein mehr als oberflächliches Deutsch lernen. Und das wird wohl nur gelingen, wenn wir, trotz allem, so etwas wie Freude oder gar Spaß am Lernen unserer Sprache vermitteln können.

Aber dies ist noch nicht die ganze Wahrheit. Mir scheint, daß dieser allgemein richtige Anspruch mit einigen Differenzierungen und Weiterentwicklungen der aktuellen Praxis sehr wohl vereinbar ist, ja sie erforderlich macht.

Lassen Sie mich also nun konkret auf die DSH und die Praxis der Sprachausbildung eingehen: Ich begrüße es, daß eine neu formulierte Rahmenordnung verabschiedet worden ist. Vor allem drei Zielsetzungen konnten erreicht werden: Die DSH und der Deutschteil der Feststellungsprüfung sind nun einheitlich beschrieben, eine Türe ist geöffnet, die Abnahme der Prüfung auch im Ausland möglich zu machen, und die gegenseitige
Anerkennung ist verbindlich festgeschrieben (wenngleich noch nicht lückenlos verwirklicht).

Dennoch scheint mir, daß sowohl der Wortlaut als auch die konkrete Handhabung noch der Weiterentwicklung und Verbesserung bedürfen. Und diese Verbesserung darf selbstredend nicht darin bestehen, daß, wie es sich hier und da andeutet, die Prüfungsergebnisse schlechter und die Durchfallquoten höher werden. Verbesserung heißt vielmehr: bedarfs- und kundenorientierte Differenzierung und Flexibilisierung.

Das gilt zunächst für den Zeitpunkt der Prüfung: Die Prüfung definiert ein vernünftiges Sprachniveau, das aber $z u B e-$ ginn des Fachstudiums für manchen Bewerber zu hoch angesetzt ist. Der Aufenthalt in Deutschland und der Studienbetrieb selbst schulen das Sprachvermögen bekanntlich am besten. Wir sollten bereit sein, Studenten bedingt also mit Auflagen zuzulassen und dann die DSH nach einem oder nach zwei Semestern abnehmen. Dies setzt allerdings ein qualifiziertes Angebot begleitender Sprachkurse voraus.

Wir sollten auch nach akademischen Fächern und nach Ziel des Aufenthalts differenzieren. Ein Geisteswissenschaftler, der ein Vollstudium absolviert, benötigt sicher einen höheren Sprachstand als ein Physiker, der ein Jahr an einem Institut mitarbeitet, an dem die Arbeitssprache weitgehend Englisch ist. Und wer einen akademischen Abschluß anstrebt, muß ebenfalls höheren sprachlichen Ansprüchen genügen als ein zeitweiliger Programmstudent/Austauschstudent, der lediglich ein Jahr lang Vorlesungen in seinem Fachgebiet hören will. (Wer sich danach zum längeren Studium entschließt, sollte dann auch die Prüfung ablegen.)

Kurz noch einmal zur gegenseitigen Anerkennung: Ich halte es für untragbar, 
daß entgegen dem Wortlaut der Prüfungsordnung in einzelnen Fällen die Anerkennung verweigert oder an Auflagen gebunden wird. Wenn die DSH wirklich an verschiedenen Hochschulorten auf unterschiedlichem Niveau durchgeführt wird, dann darf man daraus kein Problem der ausländischen Studenten machen. Dann müssen die Hochschulen - und hier ist der FaDaF ja auch aktiv um eine wirkliche Vergleichbarkeit der Prüfungen bemüht sein.

Die Rahmenordnung definiert die Freistellungsgründe (äquivalente Prüfungen). Sie definiert sie nicht abschließend, sondern läßt der örtlichen Prüfungsordnung die Möglichkeit, weitere Freistellungsgründe festzulegen. Nach meinem Dafürhalten sollte zumindest ein weiterer Freistellungsgrund verbindlich festgeschrieben werden: Absolventen eines Germanistikstudiums sollte die Prüfung erlassen werden. Auch für Austauschstudenten im Rahmen bi- oder multilateraler Programme sollte es, wie schon weithin üblich, eine Sonderregelung geben.

Über den Tag hinaus, wenn der Wortlaut der Prüfungsordnung erneut überarbeitet wird, sollte auch ein leichter lesbarer Text entstehen. Gewiß wendet sich die Prüfungsordnung zunächst an die deutschen Fachkollegen, die Prüfer nämlich. Aber die ausländischen Studenten informieren sich auch selbst. Und für diese Leser ist der Text immer noch zu sehr mit Fachterminologie belastet. Ich schließe mich da übrigens selbst nicht aus.

Ohne Zweifel lernt man die Fremdsprache im Land der Fremdsprache am besten, dann auch eingebunden in Alltag und kulturelle Kontexte. Ich vertrete dennoch die Auffassung, daß eine möglichst weitgehende sprachliche Vorbereitung im Heimatland sinnvoll ist und daß die deutsche Seite dabei behilflich sein sollte. Wir sollten die Sprachausbildung im Heimatland und in Deutschland nicht in einen Gegensatz bringen. Es geht mir überhaupt nicht darum, Lehrkapazität in Deutschland überflüssig $\mathrm{zu}$ machen, etwa um Kosten zu sparen; sondern ich will die Ausbildung in Deutschland bereits auf mittlerem Niveau einsetzen lassen, und ich will auch den zeitlichen Lernaufwand in Deutschland für die Studierenden reduzieren.

Karrierebewußte hochqualifizierte Nachwuchskräfte, die als postgraduates auch nach USA gehen könnten, kommen nicht für ein ein-bis zweijähriges Fachstudium nach Deutschland, wenn sie das zunächst ein halbes bis ganzes Jahr Sprachausbildung in Deutschland kostet.

Wir haben ja auch im Ausland ein Lehrangebot: Die Universitäten bieten Deutsch für Hörer aller Fakultäten an, die GoetheInstitute bieten Kurse an, gelegentlich auch private Träger. Keine Berührungsangst! Wir sollten alle diese Angebote und zusätzlich die Arbeit mit Selbstlernmaterialien zur Nutzung empfehlen und dann in Deutschland auf höherem Niveau weiterqualifizieren.

Für die meisten Deutschlernenden bleibt es aber auch nach vielen Kursstunden eine offene und bange Frage, wo sie eigentlich stehen, was ihnen noch fehlt und ob sie die sprachliche Hürde vor Aufnahme des Fachstudiums schaffen werden. Der DAAD hat daher die Inititative ergriffen, zu einem einheitlichen Deutschtest nach dem Muster des TOEFL zu gelangen. Zielsetzung ist, mehr Vergleichbarkeit und Einheitlichkeit herzustellen, eine realistische Selbsteinschätzung für Bewerber und Interessenten zu ermöglichen und - dies mag kontrovers sein - bei einer definierten Punktzahl Freistellung von der DSH und damit Gewißheit auf Zulassung zu gewährleisten. Bei einem Test mit Punkteskala könnte dann auch mit verschiedenen Punktzahlen je nach Studienrichtung oder Ziel des Aufenthalts differenziert werden. Wir 
werden diese Entwicklung jetzt mit Nachdruck betreiben, und der Fachverband DaF ist herzlich eingeladen, dabei eine wichtige Rolle zu spielen. Vorbereitende Gespräche dazu haben schon begonnen.

Ein paar Worte noch zum institutionellen Rahmen der Sprachausbildung hierzulande: Ich habe schon betont, daß ich die Sprachausbildung der ausländischen Studenten zu den Aufgaben der deutschen Hochschulen zähle. Damit widerspreche ich nachdrücklich allen Bestrebungen, diese durch Privatisierung auszulagern. Ich kann dafür auch keine andere Begründung sehen, als das schlichte aber machtvolle Argument einer Kostenreduzierung. Dem allerdings müssen sich die Lehrgebiete stellen und sich marktbewußt verhalten. Wenn sie zu vergleichbaren Kosten arbeiten, gibt es keinen vernünftigen Grund für die Auslagerung der Sprachkurse. Ich halte schon die Verselbständigung der Studienkollegs für einen Fehler und ein handicap im Wettbewerb um qualifizierte Studienbewerber. Dem dürfen wir keine weiteren Komplizierungen für ein konsistentes Angebot der Hochschulen hinzufügen.

Allerdings scheinen mir die Kapazitäten für den studienbegleitenden Unterricht ganz unzureichend zu sein. An einigen Hochschulen ist das Angebot nur kläglich zu nennen. Wir sollten dringend $\mathrm{zu}$ einer Ausweitung kommen. Freilich fehlen uns detaillierte und aktuelle Zahlen, und dies nicht nur in diesem Bereich. Wir haben in den letzten Wochen daher zusammen mit dem FaDaF eine rasche Umfrage durchgeführt. Eine Auswertung wird in Kürze vorliegen. Auf dieser Basis sollten wir dann ein realistisches Konzept für die Weiterentwicklung der Sprachangebote erarbeiten. Dazu biete ich Ihnen gerne die Unterstützung des DAAD an.
Lassen Sie mich zum Abschluß in kurzer Form meine wichtigsten Thesen noch einmal zusammenfassen:

1. Für die Attraktivität der deutschen Hochschulen für hochqualifizierte ausländische Studienbewerber ist die Sprachenfrage ein Kernthema. Die Arbeit der Lehrgebiete Deutsch als Fremdsprache hat an Bedeutung gewonnen.

2. Wir müssen akzeptieren, daß Deutsch nicht mehr die weltweit wichtigste Wissenschaftssprache ist. Das heißt: wir müssen mehr werben und »kundengerechtere« Ausbildungsangebote machen.

3. Wir dürfen das Erlernen der deutschen Sprache nicht vorrangig als Problem, als Erschwernis und als Hürde sehen, sondern als lohnende Lernund Lebenserfahrung »anbieten «. Wir müssen uns aber fragen, ob die Sprach-Lehrangebote dieser Zielsetzung schon gerecht werden.

4. Intensive sprachliche Vorbereitung im Heimatland - auch mit neuen Medien - steht nicht im Gegensatz zu qualifizierter Sprachausbildung in Deutschland. Für den Aufenthalt in Deutschland steht immer nur begrenzte Zeit zur Verfügung. Die Sprachausbildung hierzulande sollte bereits auf mittlerem oder höherem Niveau aufsetzen können.

5. Es sollte ein einheitlicher »Test-DaF« eingerichtet werden. Der Gewinn wäre eine Differenzierungsmöglichkeit gemäß einer Punkteskala, eine realistische Selbsteinschätzung für Bewerber und eine Gewißheit auf Zulassung bei Erreichen einer definierten Punktzahl.

6. Die Anforderungen bei der DSH sollten je nach Studienzweck und Fach differenziert und flexibilisiert werden. Kriterien wären: Vollstudium oder Teilstudium, Erststudium oder Auf- 
bau-/ Promotionsstudium, fachspezifische Anforderungen. Eine bedingte Zulassung mit der Auflage einer DSH-Prüfung zu einem späteren Zeitpunkt sollte ermöglicht werden.

7. Die DSH-Prüfungsordnung muß auch für ausländische Interessenten verständlich werden. Die Anforderungen müssen kalkulierbar sein. Die Prüfungsbeispiele dürfen nicht abschrekkend sein. Diese Forderungen scheinen mir noch nicht hinreichend erfüllt.

8. In diesem Rahmen gewinnen die studienbegleitenden Sprachkurse erhöh- te Bedeutung. Sie sollten dringend ausgeweitet werden. Eine genaue empirische Analyse der aktuellen Situation ist noch $\mathrm{zu}$ leisten.

9.Sprachliche Ausbildung gehört $\mathrm{zu}$ den Aufgaben der Hochschulen und sollte nicht privatisiert und ausgegliedert werden. Ein kundengerechtes und kostengünstiges Angebot muß auch innerhalb der Hochschulen möglich sein.

10. In diesem Sinne bitte ich Sie um Mithilfe und biete meinerseits die Unterstützung des DAAD für gemeinsame Anstrengungen an. 